\title{
Modelo de Predicción de Costos en Servicios de Salud Soportado en Simulación Discreta
}

\author{
Jhonathan M. Vargas y Jaime A. Giraldo \\ Facultad de Ingeniería y Arquitectura, Universidad Nacional de Colombia, Sede Manizales. Manizales, \\ Caldas - Colombia (e-mail: jmvargasba@unal.edu.co y jaiagiraldog@unal.edu.co)
}

Recibido Dic. 16, 2013; Aceptado Mar. 3, 2014; Versión final recibida Mar. 28, 2014

\begin{abstract}
Resumen
Se ha desarrollado un modelo de apoyo a la toma de decisiones sobre los costos de prestación de servicios de salud. Se usa simulación discreta, evaluando diferentes escenarios de prestación de servicios de una Entidad Prestadora de Servicios de Salud (EPS): consulta médica general, urgencias, hospitalización y enfermedades catastróficas, entre otros, El modelo se realizó para que una EPS típica simule el costo de prestación de servicios por un año, a partir de indicadores tomados de estadísticas de salud del orden nacional cuyos valores fueron ajustados al comportamiento específico de la EPS. Los resultados obtenidos con el modelo permitieron determinar que el enfoque de cálculo de costos es efectivo, y que los promedios nacionales de ciertos parámetros deben ser modificados para cada caso. De otra forma se pueden producir errores importantes en el presupuesto al no incluir la variación en estos parámetros.
\end{abstract}

\section{Prediction Model of Costs in Health Services Supported in Discrete Simulation}

\begin{abstract}
A model to support decision making about health service is proposed. Discrete simulation has been used, evaluating different scenarios of health service delivered by an Institution of Health Services (IHS): general medical consultation, emergency, hospitalization and catastrophic diseases, among others. The model was developed so any IHS can simulate typical cost of service for one year using indicators from national health statistics adjusting values to the specific behavior of the HIS. The results obtained with the model have revealed that the costing approach is effective, and that the national average values of certain parameters must be modified for each case. Otherwise, important errors can be introduced in the budget when the variations of these parameters are not included.
\end{abstract}

Keywords: discrete simulation, decision support, health services, health care costs 


\section{INTRODUCCIÓN}

En los últimos años, la crisis en el sector salud en Colombia se ha incrementado, cientos de pacientes deben hacer largas filas para la atención desde tempranas horas de la mañana, demasiadas quejas con respecto a demoras en la atención, muchos servicios no pueden ser prestados por problemas en la planificación/asignación de recursos, alta incertidumbre en el costo total del servicio, entre otros, son algunos de los problemas que deben enfrentar los usuarios/gerentes a la hora de requerir/prestar atención en salud. No es un secreto la difícil situación financiera que atraviesa el sector salud colombiano, aunado a una tendencia creciente en la población de adultos mayores con patologías de alta complejidad que implican un alto costo aumentando los riesgos financieros, según estadísticos propios de una típica E.P.S. colombiana, (Unisalud, 2012).

Se considera la asistencia en salud como un conjunto de fenómenos que ocurren en un contexto organizacional. El aspecto fundamental de este campo es que los pacientes son los que buscan tratamiento de un doctor por una patología. Los médicos son típicamente trabajadores individuales o empleados de un hospital. Los pacientes, por otra parte, son empleados a los cuales sus empleadores les cancelan un seguro con el cual deben pagar los servicios médicos. Así, los dos contextos organizacionales inmediatos son el cuidado de la salud como una organización proveedora y pagadora (Basole et al., 2013). Los sistemas de salud se enfrentan a retos, incluyendo la disminución de los recursos y el aumento de la demanda de servicios. Estos retos tienen que ser equilibrados en este complejo sistema de sistemas para asegurar una calidad de vida sostenible, que debe considerar las necesidades de las generaciones futuras sin comprometer las necesidades de las generaciones actuales. El componente social de la sostenibilidad es una de las áreas importantes de la sostenibilidad de la salud. El componente social se centra en consideraciones tales como la equidad, el empoderamiento, la accesibilidad, la participación, la identidad cultural y la estabilidad institucional. La satisfacción del paciente es un factor clave en el elemento social en términos de costo, accesibilidad a los servicios y recursos, y el bienestar del paciente (Faezipour y Ferreira, 2013).

El sistema de salud colombiano hace parte del sistema de seguridad social de Colombia el cual es regulado por el gobierno nacional en dos regímenes de afiliación al sistema: contributivo y subsidiado. Con respecto a los servicios prestados tanto por el régimen contributivo como por el régimen subsidiado, la ACEMI (Asociación Colombiana de Empresas de Medicina Integral) presenta una recopilación estadística que muestra cómo se han distribuido los servicios prestados, Camacho Rojas (2011). Los sistemas de atención a pacientes se caracterizan por exhibir en su operación alta variabilidad (aleatoriedad), presentan múltiples interacciones entre: pacientes, equipo médico, prestadores y otros recursos, lo cual aumenta su grado de complejidad, dado su comportamiento dinámico. Dicha complejidad es difícil modelarla con matemática analítica, Kelton y Law (2007), por lo que en este estudio se propone el empleo de técnicas de simulación discreta que recrean el comportamiento del sistema de forma más precisa (incluyendo la variabilidad) y acorde con la realidad, al incluir las interdependencias entre los principales componentes del sistema.

En una revisión preliminar de la literatura, autores como Thorwarth y Arisha (Thorwarth y Arisha, 2012), Anthony Virtue, Thierry Chaussalet John Kelly (2011) han trabajado la simulación para sistemas de salud, específicamente en la atención de pacientes. La simulación en sistemas de salud es un área, aunque nueva, muy activa en investigación en aspectos operativos de estos sistemas. Una de las primeras aplicaciones de la simulación en sistemas de salud se dio hace más de 30 años. England y Roberts (1978), reportan el uso de 92 modelos y dan una idea de la historia de la simulación como enfoque de modelado de sistemas de cuidados de la salud y el esfuerzo para aplicar los modelos de simulación, a pesar de la limitación en los equipos de cómputo en dicha época. Sin embargo, parece que en los años 1960 y 1970 , son pocos los estudios que informaron de cualquier uso con éxito de los modelos y esto no significa que el flujo haya cesado. La simulación de eventos discretos ha sido ampliamente usada en el modelamiento de sistemas de salud, siendo esta una de las herramientas y métodos usados en el análisis y mejora de sistemas de salud (Günal y Pidd, 2010). Gupta y Sharda (2013) identifican seis áreas clave de investigación en el dominio de la salud que reconocen como corrientes importantes para la realización de más investigaciones y que pueden tener un enorme impacto en la mejora del proceso de prestación de servicios de salud y resultados asociados, proponiendo un marco de trabajo para usar enfoques de modelamiento de sistemas de cuidados de la salud basados en análisis de datos y simulación.

A primera vista, mucho de lo que hace tan atractivo el modelado de los sistemas de salud como una herramienta de análisis de decisiones en la atención de pacientes, es exactamente lo que crea barreras en algunos médicos y profesionales de la salud. El concepto de que las matemáticas pueden ser empleadas para modelar la realidad es difícil de comprender para el típico médico. Estos son entrenados fuertemente en las ciencias biológicas de las cuales la mayoría de las matemáticas son removidas. El primer paso en la desmitificación de los modelos a los ojos de los médicos es aclarar que estos modelos pueden ser 
simplemente para descomponer la realidad modelada en trozos pequeños y generar confort en soluciones de "súper-humanos" a los problemas "sencillos" (Fackler y Speader, 2011).

Se reconoce cada vez más que los sistemas de cuidados de la salud son complejos, con recursos limitados y muchas fuentes de interacciones de retroalimentación tanto positiva como negativa. La simulación de eventos discretos es una herramienta que acomoda fácilmente las cuestiones de planificación de la capacidad, la gestión del rendimiento y la interacción de recursos (Ramwadhdoebe et al. 2009). Los sistemas de salud tienen características específicas para su modelamiento. En estos sistemas los tiempos de servicio son especialmente más largos que en otros sistemas de servicios, los clientes requieren ser identificados antes que el servicio sea provisto, pudiendo estos salir del sistema y retornar y el costo generalmente es determinado después que el servicio es prestado (Giraldo Garcia, 2012). Al momento de modelar y simular sistemas de salud se encuentran barreras que se concentran en las partes interesadas, las herramientas utilizadas y la complejidad dada del sistema. Las barreras que presenta el modelamiento citadas en la literatura pueden ser categorizadas en tres clases de barreras: conflicto de intereses, falta de herramientas relevantes y expectativas no coincidentes (Eldabi, 2009).

El uso de modelos de simulación en la salud basado en herramientas de soporte a decisiones apunta a una cuestión fundamental, la salud es un campo complejo, que requiere un conocimiento detallado sobre (Basole et al., 2013):

i) Fenómenos médicos, intervenciones y progresión sobre el tiempo.

ii) Procesos organizacionales e inter-organizacionales que involucran entre otras cosas flujo de información y de pacientes.

iii) Costos, reembolsos y reglas de capitación, y relaciones entre costos y resultados en la salud.

iv) El efecto de los incentivos y la información sobre el comportamiento de actor individual y el rendimiento general del sistema.

Entre las barreras naturales encontradas al momento de modelar y simular los sistemas de salud se cuenta entre otras que estos no tienen regla de parada (ya que generalmente operan $7 \times 24$ ), cada modelo es esencialmente único, cada problema puede ser considerado un síntoma de otro problema y cada problema puede ser explicado de diferentes maneras y esta explicación determina la naturaleza de la solución. Estos son algunos retos a los que se enfrentan los modeladores al momento de concebir un modelo que represente la realidad. La adopción de técnicas de modelamiento y simulación en los sistemas de salud se ha visto en aumento aunque en menor proporción que otros sectores como el sector de negocios, aeroespacial y el sector comercial. Para Young et al (2009), el sector salud se enfrenta a tres retos críticos en este sentido: el primero es la pregunta ¿el modelamiento y la simulación son suficientemente buenos?, el segundo es el vínculo del modelamiento con la toma de decisiones y el tercero es la cultura necesaria para sacar el mayor provecho del modelamiento y la simulación. Sobre los dos primeros aspectos se centra este trabajo.

Para que los profesionales de la medicina puedan comprender mejor los modelos, es necesario que vean a dichos modelos como sistemas simples de entrada-salida, así se facilitara su comprensión y estos podrán ser utilizados con mayor frecuencia en los sistemas de salud como herramienta en toma de decisiones en diferentes áreas. Generalmente estos profesionales operan sistemas de salud que se caracterizan por su alta complejidad, en términos de volúmenes de datos y variables interdependientes. En estos casos es necesario contar con herramientas que ayuden a la toma de decisiones requerida por el sistema de salud estudiado (Fackler y Speader, 2011). Desde el punto de vista del administrador de salud, la eficiencia se mide a menudo por el costo de proporcionar un determinado nivel de servicio. Los usuarios de los servicios de salud, por otro lado, además de exigir una buena calidad de servicio, desean una pronta atención, (De Angelis et al. 2003).

En Colombia, las TI (Tecnologías de Información) en salud han sido incorporadas con progresivo éxito por parte de las organizaciones y entidades responsables de la salud pública, ((Fonseca Cruz, García Gracia, Mejía Medina, Marin Cortes, \& Bejarano Sandoval, 2013). De hecho, en 2013 fue lanzado el SISPRO, Sistema Integral de Información de la Protección Social, el cual produce, organiza y estructura información con el fin de gestionar el conocimiento y divulgar resultados que permitan avanzar hacia mejores decisiones en salud, según lo expresa la Dirección de Epidemiología y Demografía - DED del Ministerio de Salud y Protección Social (MSPS). En un contexto real de una E.P.S. en el ámbito colombiano, es posible hoy gracias al empleo de TI mantener registro histórico de datos de varios años sobre servicios prestados, costos incurridos y caracterización de la población beneficiaria, por lo que se propone el modelo de decisión mostrado en la expresión (1) y cuyos componentes de E/S se describen en la Tabla 1, teniendo en cuenta que la expresión (2) es la limitante de los porcentajes de servicios, la cual no debe superar el 100\%, y la expresión (3) es condición del sistema donde las distribuciones porcentuales de los servicios no pueden ser 
menores a 0 ni mayores a 100. En el caso de servicios correspondientes a alto costo, estos son variables y comprenden patologías como cáncer, infección por VIH, grandes quemaduras, insuficiencia renal crónica, patologías del sistema nervioso central, patologías cardiacas, reemplazos auriculares, uso de unidad de cuidado intensivo entre otros y cuyo costo puede variar entre 1.000 USD y 10.000 USD. Los usuarios frecuentes son aquellos que en promedio en el año utilizan una significativa cantidad de servicios, mientras que los normales utilizan una menor cantidad, siendo los límites y la categorización de pacientes frecuentes/normales propios de cada E.P.S

$y=f\left(x_{1}, x_{2}, x_{3}, x_{4}, x_{5}, x_{6}, x_{7}, x_{8}, x_{9}\right)$

Dónde:

Tabla 1. Componentes de entrada/salida del modelo de decisión en costo.

\begin{tabular}{|c|l|}
\hline Variables & \multicolumn{1}{c|}{ Descripción de la Variable } \\
\hline$x_{1}$ & \% de servicios correspondiente a Consulta médica Gral. \\
\hline$x_{2}$ & \% de servicios correspondiente a Otros Ambulatorios \\
\hline$x_{3}$ & \% de servicios correspondiente a Urgencias \\
\hline$x_{4}$ & \% de servicios correspondiente a Hospitalario \\
\hline$x_{5}$ & \% de servicios correspondiente a Partos y cesáreas \\
\hline$x_{6}$ & \% de servicios correspondiente a Domiciliario \\
\hline$x_{7}$ & \% de servicios correspondiente a Alto Costo \\
\hline$x_{8}$ & Numero de servicios correspondiente a un paciente frecuente \\
\hline$x_{9}$ & Numero de servicios correspondiente a un paciente normal \\
\hline$y$ & Variable respuesta, para el modelo representa los costos totales \\
\hline$f\left(x_{i}\right)$ & Modelo de predicción \\
\hline
\end{tabular}

$\sum_{i=1}^{7} x_{i}=100$

$0>x_{i}<100 \quad\{i \in N: 1 \leq i \leq 7\}$

$0>x_{i}<\operatorname{Max} \quad\{i \in N: 8 \leq i \leq 9\}$

El desarrollo matemático de la función $f$ expresada en (1) presenta grandes retos, si el sistema real que se está modelando no se comporta linealmente e incluye variables aleatorias, como es el caso de los sistemas de salud. Surge una pregunta importante en este punto: ¿Cómo representar apropiadamente un sistema de salud dada su alta interdependencia entre sus componentes y aleatoriedad, en pro de no perder realismo y poder medir algunas salidas de interés a partir de unos factores de entrada? Como lo describe Shannon (1988), en esta representación interesan tres componentes distintos: la entrada, el sistema y la respuesta. Para modelar un componente, se debe tener disponible o suponer el conocimiento acerca de dos de los tres. Si se conocen las ecuaciones que describen el comportamiento del sistema dinámico, entonces el problema directo es encontrar la respuesta del sistema para cada entrada determinada. De otro lado, si se conocen las ecuaciones que describen el sistema y se especifica o requiere una respuesta, el problema de encontrar las entradas necesarias para producir dicha respuesta es clasificado como un problema de control. El problema más difícil surge cuando se da un conjunto de entradas y salidas correspondientes de un sistema, y se tiene que derivar o encontrar una descripción matemática del sistema. Este último tipo de problema se conoce como el problema de identificación de la estructura. De lo anterior queda claro que el problema de modelar un sistema de salud puede caer en la categoría de identificación de la estructura, en la cual el proceso de prestación de servicios es estocástico, en razón a la indeterminación de la salida para una entrada dada.

Al aproximarse a la clase de modelos que se requiere para representar y dado por la expresión (1), Kelton y Law (2007), Banks et al. (2009) y Shannon (1988) están de acuerdo en que se ha de considerar el modelamiento de sistemas complejos (como los de cuidados de la salud) mediante simulación si: i) No existe una formulación matemática del problema; ii) Las técnicas analíticas están disponibles, pero los 
procedimientos matemáticos son tan complejos y difíciles, que la simulación proporciona un método más simple de solución; iii) Se desea observar el comportamiento simulado del sistema sobre un periodo de tiempo; y iv) Se requiere la aceleración del tiempo para sistemas que exigen de largo tiempo para realizarse.

Se plantea entonces un modelo soportado en simulación discreta como herramienta para la toma de decisiones respecto al costo de usar y asignar recursos en una unidad de servicios de salud. En dicho modelo se permite al usuario entrar dos valores (un mínimo y máximo) de las variables de decisión $x_{1}, x_{2}, x_{3}, x_{4}, x_{5}, x_{6}, x_{7}, x_{8}, x_{9}$ permitiéndole representar hasta $2^{9}$ diversos escenarios posibles de estimación de costos.

\section{DESARROLLO DEL MODELO DE APOYO A DECISIONES EN COSTO DE SALUD}

El modelo de soporte a decisiones desarrollado simula el comportamiento de los servicios prestados y la cantidad de servicios requerida por pacientes normales y frecuentes, por una E.P.S. típica para un periodo de tiempo dado, y como resultado proporciona los costos asociados, según se muestra en la Figura 1.

El flujo en detalle, representado en la Figura 1 es el siguiente: por cada día del año se simula la llegada de pacientes; por cada paciente, el modelo muestrea el tipo de servicio según una distribución de probabilidad empírica, acorde al servicio se muestrea el costo del mismo y finalmente se clasifica el paciente como normal o frecuente empleando distribuciones de probabilidad uniformes a fin de determinar cuántos servicios utilizara este en el año, mediante los parámetros DSPN (distribución numero servicios paciente normal) y DSPF (distribución número de servicios paciente frecuente). Al terminar de simular el año, el modelo devuelve los costos totales.

Para la simulación se utilizó el software MEDMODEL (Promodel, 2014), el cual es un simulador con animación para computadoras personales, especializado en simulación de sistemas de salud. Una representación gráfica del modelo se muestra en la Figura 2.

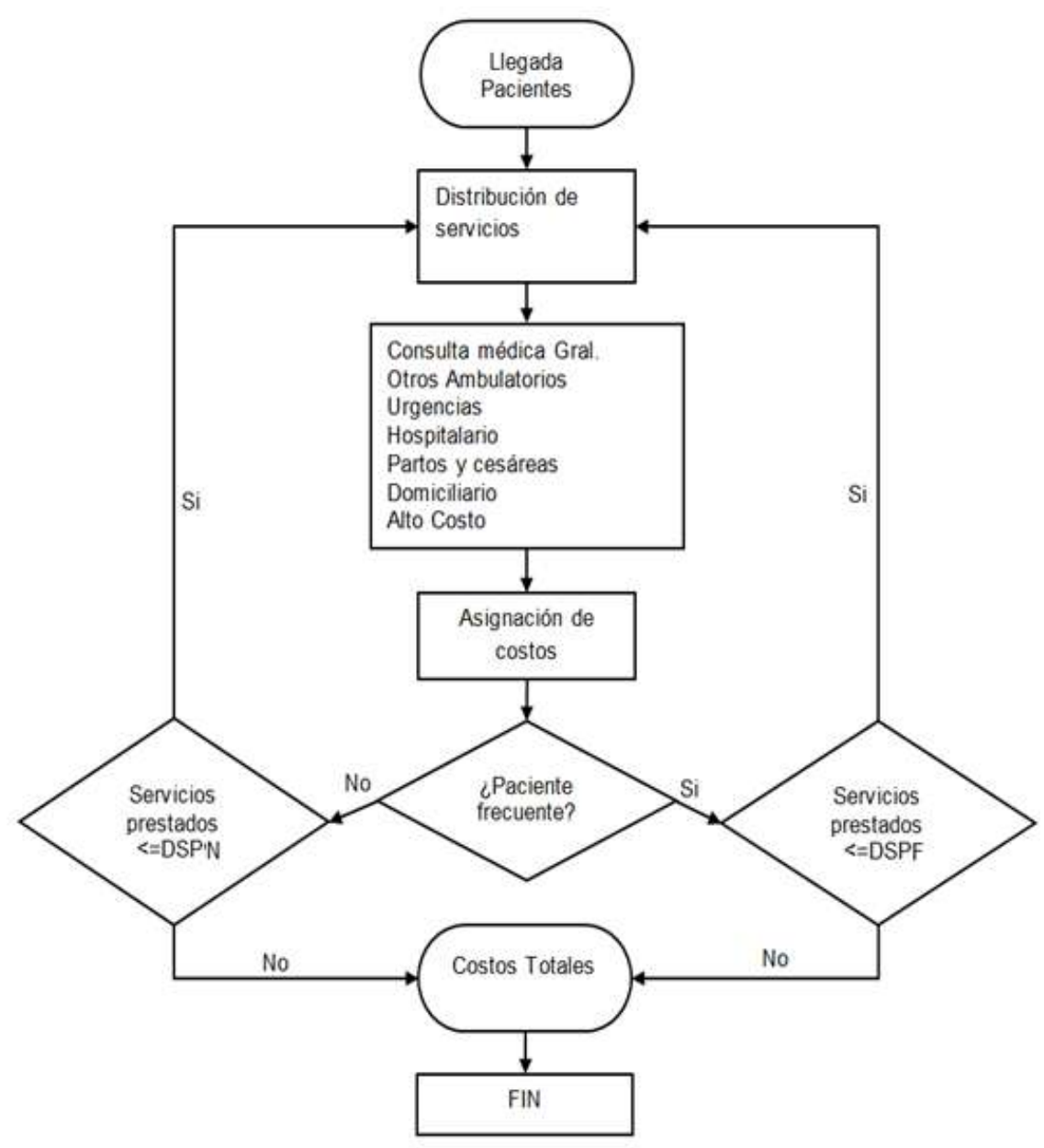

Fig. 1. Diagrama del modelo de soporte a decisiones en costo. 


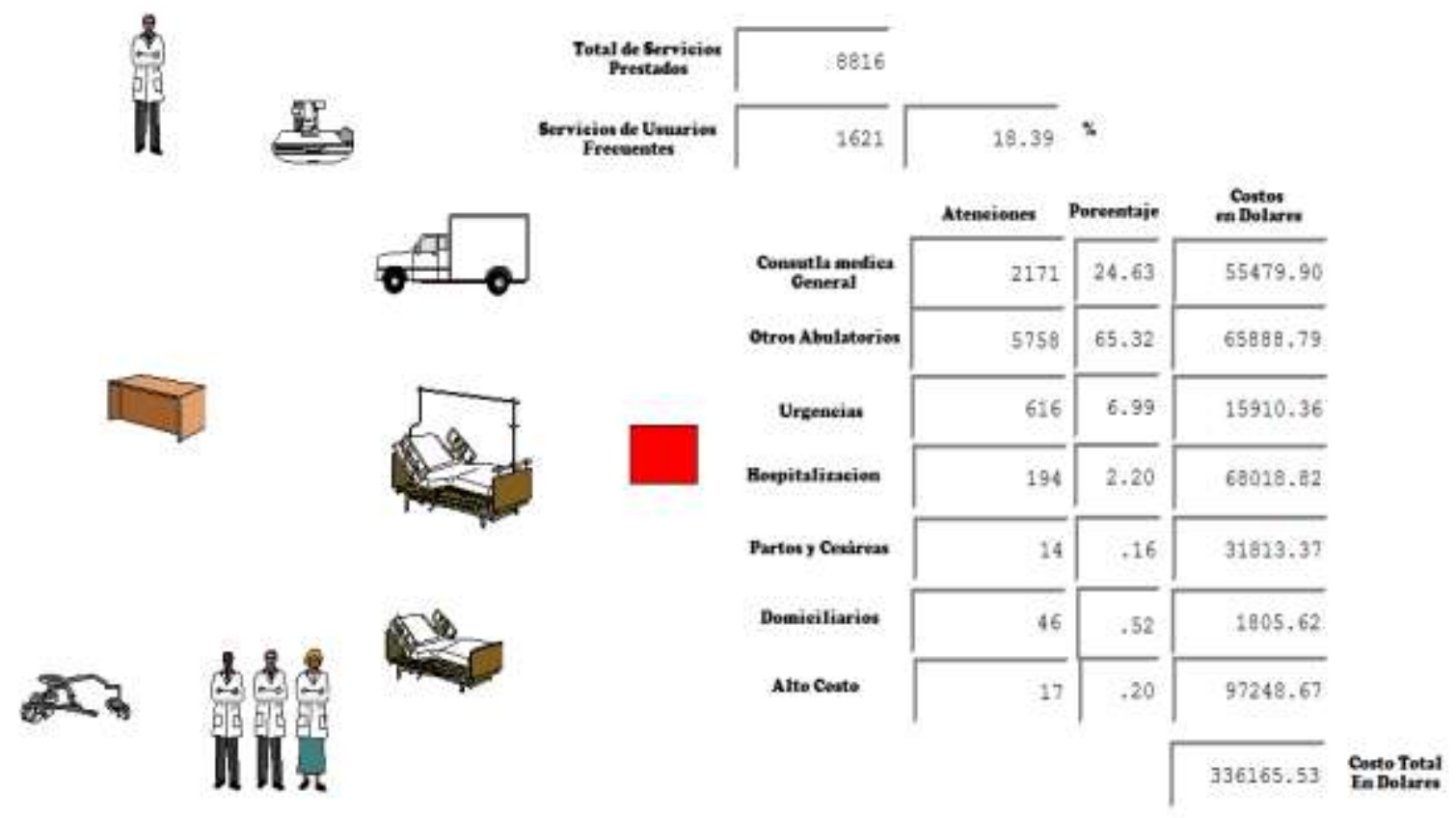

Fig. 2. Representación gráfica del modelo de simulación.

El modelo de simulación desarrollado, dispone de una interfaz con el software Excel, mediante la cual el usuario puede establecer los valores para las variables de decisión: $x_{1}, x_{2}, x_{3}, x_{4}, x_{5}, x_{6}, x_{7}, x_{8}, x_{9}$. Estos valores se deben proporcionar entre un mínimo y un máximo, pues el modelo los muestrea según una distribución de probabilidad uniforme.

Igualmente es posible con los resultados de costo, efectuar análisis de varianza (ANOVA) a fin de determinar estadísticamente que variables de decisión tienen un impacto importante en el costo. Dado el alto volumen de escenarios posibles $\left(2^{9}\right)$, se sugiere emplear diseños fraccionados, según se considera en diseño experimental $2^{k}$, (Montgomery 2004).

\section{RESULTADOS Y DISCUSIÓN}

Con el fin de probar el modelo de simulación se utilizaron datos reportados por el sector salud en Colombia, tanto a nivel nacional como a nivel de una E.P.S. en particular. Para simular los posibles valores de las variables de decisión $x_{1}, x_{2}, x_{3}, x_{4}, x_{5}, x_{6}, x_{7}$, se emplearon los datos reportados por la ACEMI (Camacho Rojas, 2011), los cuales corresponden a una distribución porcentual de los servicios prestados por las E.P.S. del régimen contributivo colombiano y una proyección al año 2013 de la tasa de servicios prestados, basada en índices de crecimiento demográfico, según se muestra en la Tabla 2.

Tabla 2. Datos proyectados de los servicios prestados en salud a 2013 por el régimen contributivo en Colombia, elaboración propia basados en Camacho Rojas (2011)

\begin{tabular}{|c|c|c|c|}
\hline Servicio & Porcentaje & Servicios & Costos Estimados \\
\hline Consulta médica General & $24,79 \%$ & 47.925 .350 & 1.068.155.408 USD \\
\hline Otros Ambulatorios & $64,99 \%$ & 125.643 .274 & 1.236.455.459 USD \\
\hline Urgencias & $7,17 \%$ & 13.861 .727 & 34.098.462 USD \\
\hline Hospitalario & $2,27 \%$ & 4.396 .709 & 1.386.315.323 USD \\
\hline Partos y cesáreas & $0,15 \%$ & 288.629 & 641.678 .261 USD \\
\hline Domiciliario & $0,45 \%$ & 852.161 & 28.698.226 USD \\
\hline Alto Costo & $0,18 \%$ & 353.392 & 1.819.516.017 USD \\
\hline Total & $100,00 \%$ & 193.321 .435 & 6.214.917.157 USD \\
\hline
\end{tabular}


Respecto a los datos requeridos para simular las variables de decisión $x_{8}$ y $x_{9}$ se emplearon los reportados por la E.P.S. UNISALUD en su informe de gestión del 2012 (Unisalud, 2012) en el cual se definen los usuarios frecuentes como aquellos usuarios que en promedio en el año utilizaron entre 6 y 8 servicios y los pacientes normales hicieron uso de 5 servicios o menos. Respecto a la cantidad de servicios prestados por año, UNISALUD reportó para el 2012 un total de 7910 servicios para una población aproximada de 1800 pacientes, según el mencionado informe (Unisalud, 2012).

Tabla 3. Costos unitarios por servicio de Salud proyectados a 2013. Elaboración propia basados en datos de la Superintendencia Nacional de Salud (2010)

\begin{tabular}{|l|l|}
\hline Servicio & Costo por Servicio en USD \\
\hline Consulta médica General & entre 21,82 USD y 26,67 USD \\
\hline Otros Ambulatorios & entre 9,63 USD y 11,77 USD \\
\hline Urgencias & entre 24,08 USD y 29,43 USD \\
\hline Hospitalario & entre 308,73 USD y 377,34 USD \\
\hline Partos y cesáreas & entre $2.176,87$ USD y $2.660,62$ USD \\
\hline Domiciliario & entre 32,97 USD y 40,303 USD \\
\hline Alto costo & entre 944,49 USD y $10.258,72$ USD \\
\hline
\end{tabular}

En relación con los datos requeridos para simular los costos por servicio, se emplearon datos reportados por la Superintendencia de Servicios de Salud para el año 2010, y actualizados al año 2013 mediante el IPC (Índice de Precios al Consumidor) de 2011 y 2012, según se muestra en la Tabla 3 (Superintendencia Nacional de Salud, 2010). Dado que la Superintendencia de salud reporta valores promedios, se considera más real simularlos en un rango con variación del $10 \%$ respecto al promedio. Los servicios de alto costo dependen mucho de las patologías y por esto sus costos presentan un comportamiento aleatorio que va desde un valor mínimo de 944,49 USD para otras patologías y un valor máximo de 10.258,7195 USD para Unidad de Cuidados Intensivos, pasando por patologías como el VIH/Sida, las insuficiencias renales, cáncer entre otras.

Para simular el comportamiento de los costos totales de una típica E.P.S. colombiana y mostrar al lector las bondades del modelo de predicción propuesto, se plantean cuatro escenarios en los cuales se hace variar una vez cada variable, ver Tabla 4. El escenario 1 establece los niveles de las variables de decisión según la demanda proyectada en la Tabla 2 y numero de servicios por paciente normal y frecuente según reporte de UNISALUD (2012), siendo este escenario el referente para la comparación de las respuestas que arroja el modelo frente a diferentes cambios en las variables de decisión. En el escenario 2, se realizó una variación en la distribución de los servicios, se mantuvo el resto de variables de decisión en condiciones normales de funcionamiento, según datos reportados por UNISALUD (2012) esto con el fin de comparar los datos proyectados para el año 2013 con una pequeña variación en los servicios prestados. Para el escenario 3 se conservaron las distribuciones iniciales porcentuales de los servicios prestados, el cambio fundamental de este escenario con respecto al primero es el indicador de numero de servicios año por paciente frecuente entre 11 y 15 . Igualmente, en el escenario 4 se conservaron las distribuciones iniciales porcentuales de los servicios prestados, el cambio fundamental de este escenario con respecto al primero es el indicador de numero de servicios año por paciente normal entre 3 y 7 .

Tabla 4. Escenarios de simulación

\begin{tabular}{|c|l|r|r|r|r|}
\hline $\begin{array}{c}\text { Variables } \\
\text { decisión }\end{array}$ & \multicolumn{1}{|c|}{ Descripción de la Variable } & Escenario 1 & \multicolumn{1}{|c|}{ Escenario 2 } & \multicolumn{1}{c|}{ Escenario 3 } & \multicolumn{1}{c|}{ Escenario 4 } \\
\hline$x_{1}$ & Consulta médica Gral. & $24,8 \%$ & $30,00 \%$ & $24,8 \%$ & $24,8 \%$ \\
\hline$x_{2}$ & Otros Ambulatorios & $64,99 \%$ & $60,00 \%$ & $64,99 \%$ & $64,99 \%$ \\
\hline$x_{3}$ & Urgencias & $7,17 \%$ & $5,00 \%$ & $7,17 \%$ & $7,17 \%$ \\
\hline$x_{4}$ & Hospitalario & $2,27 \%$ & $2,50 \%$ & $2,27 \%$ & $2,27 \%$ \\
\hline$x_{5}$ & Partos y cesáreas & $0,15 \%$ & $1,00 \%$ & $0,15 \%$ & $0,15 \%$ \\
\hline$x_{6}$ & Domiciliario & $0,45 \%$ & $0,75 \%$ & $0,44 \%$ & $0,44 \%$ \\
\hline$x_{7}$ & Alto Costo & $0,18 \%$ & $0,75 \%$ & $0,18 \%$ & $0,18 \%$ \\
\hline$x_{8}$ & $\begin{array}{l}\text { Numero servicios año paciente } \\
\text { frecuente }\end{array}$ & Entre 8 y 10 & Entre 8 y 10 & Entre 11 y 15 & Entre 8 y 10 \\
\hline$x_{9}$ & $\begin{array}{l}\text { Numero servicios año paciente } \\
\text { normal }\end{array}$ & Entre 3 y 7 & Entre 3 y 7 & Entre 3 y 10 & Entre 3 y 7 \\
\hline
\end{tabular}


Al experimentar mediante el modelo de simulación en cada escenario, en la Tabla 5 se muestra el costo total obtenido empleando la simulación para un periodo de tiempo de servicios de 1 año, y 10 réplicas en cada escenario simulado. Igualmente para propósitos de comparación se muestra en esta tabla adicionalmente el costo total empleando valores promedios (ver Tabla 3 ) de los servicios y sus respectivas variaciones respecto al valor simulado. Como puede apreciarse, la predicción del costo total para una pequeña E.P.S. mediante el empleo de promedios puede llegar a tener variaciones de más de 150 millones de pesos con respecto a la predicción con simulación y porcentualmente hasta del $8.7 \%$. Esta variación puede representar una cantidad significativa de dinero, si por ejemplo, se simulara una E.P.S. como Nueva E.P.S. que tiene aproximadamente 100 veces el tamaño de UNISALUD.

Tabla 5. Prediccion de costos totales

\begin{tabular}{|c|c|c|c|c|c|}
\hline Escenario & $\begin{array}{c}\text { Costo estimado } \\
\text { por simulación }\end{array}$ & $\begin{array}{c}\text { Numero de } \\
\text { servicios } \\
\text { prestados }\end{array}$ & $\begin{array}{c}\text { Costo estimado por } \\
\text { promedio }\end{array}$ & Variación en USD & Variación en \% \\
\hline 1 & 336.166 USD & 8.816 & 322.225 USD & 13.941 USD & 4,1 \\
\hline 2 & 869.747 USD & 8.816 & 794.207 USD & 75.540 USD & 8,7 \\
\hline 3 & 183.774 USD & 4.940 & 180.557 USD & 3.217 USD & 1,8 \\
\hline 4 & 658.805 USD & 18.293 & 668.610 USD & -9.805 USD & $-1,5$ \\
\hline
\end{tabular}

Finalmente, el modelo desarrollado permite hacer análisis por comparación de escenarios de operación del sistema. Así por ejemplo, es de resaltar que al comparar el Escenario 1 con el Escenario 2 en la Tabla 5, se puede inferir que los servicios que influencian mayormente los costos son las enfermedades catastróficas que se asocian a servicios de alto costo, así un aumento de 4 veces la cantidad de servicios de alto costo representó un aumento de casi 3 veces los costos totales.

\section{CONCLUSIONES}

De los resultados expuestos, de su cálculo/análisis y discusión, se pueden obtener las siguientes conclusiones, sobre el modelo de predicción de costos en salud:

La cualidad más importante del modelo es poder representar la alta variabilidad e interdependencia que los sistemas de cuidados de la salud exhiben en su operación. Como pudo comprobarse, esta variabilidad se modelo mediante el diseño de escenarios de operación en términos de variables de decisión, cuyos valores se establecen entre un mínimo y un máximo. Y la interdependencia se representó mediante un modelo de simulación discreta que mide el desempeño del sistema en términos de costo.

La aplicación del modelo puede ser realizada fácilmente por una E.P.S. si esta mantiene registro informático histórico de datos de varios años sobre servicios prestados, costos incurridos y caracterización de la población beneficiaria. A partir del conocimiento de estos datos, el tomador de decisiones establece los escenarios de simulación más convenientes a sus propios objetivos.

Al realizar una prueba piloto del modelo en una E.P.S. típica y medir/comparar el costo simulado vs costo promediado se encontró que la predicción del costo total mediante el empleo de promedios puede llegar a tener altas variaciones en valor y porcentaje respecto a la predicción con simulación, dentro de intervalos estadísticamente aceptables.

\section{AGRADECIMIENTOS}

Los autores desean dar reconocimiento a la Universidad Nacional de Colombia Sede Manizales, UNISALUD y su departamento de Ingeniería Industrial. Igualmente se agradecen los aportes dados en Valderrama (2005) sin los cuales no se hubiera podido redactar este informe.

\section{REFERENCIAS}

Banks J., Carson, J., Barry, L. N., Nicol, D.; Discrete-Event System Simulation., 4a Edicion, Prentice Hall, Nueva York, (2009).

Basole, R. C., Bodner, D. A., y Rouse, W. B.; Healthcare management Through Organizational Simulation. Desicion Support System, 552-563, (2013). 
Camacho, S. M.; Cifra y Datos de Servicio de Salud ACEMI. Bogotá, (2011).

De Angelis, V., Felici G., Impelluso P.; Integrating Simulation and Optimisation in Health Care Centre Management, European Journal of Operational Research, 101-114, (2003).

Eldabi, T.; Implementation issues of Modeling Healthcare Problems: Misconceptions and lessons. Winter Simulation Conference, 1831-1839. Austin, (2009).

England W. y Roberts S.; Applications of computer simulation in health care. Winter Simulation Conference, (1978).

Fackler, J., y Speader, M.; Why Doesn't Healthcare Embrace Simulation and Modeling? What Would It Take? Winter Simulation Conference, 1137-1142. Phoenix, (2011).

Faezipour, M., Ferreira, S.; A System Dynamics Perspective of Patient Satisfaction in Healthcare, Procedia Computer Science, 148-156, (2013).

Fonseca, J., García Gracia, C. A., Mejía Medina, F., Marín Cortes, S., \& Bejarano Sandoval, A.; Las Tic En Los Retos Del Sector Salud - Volumen 2. Bogotá: Colombia Digital, (2013).

Giraldo, J. A; Simulación de Sistemas de Produccion y de Servicios. Manizales: Editorial UN, (2012).

Günal, M., y Pidd, M.; Discrete Event Simulation for Performance Modelling in Healthcare: a Review of the Literature. Journal of Simulation, 42-51, Lancaster, (2010).

Gupta, A., Sharda, R.; Improving the Science of Healthcare Delivery and Informatics Using Modeling Approaches. Decision Suport System. 423-427. (2013).

Kelton, W. D., y Law, A. M.; Simulation Modeling and Analysis 3nd Ed. McGraw-Hill, (2007).

Ministerio de Salud y Protección Social., Ministerio de Salud y Protección Social, Régimen Contributivo. Obtenido de http://www.minsalud.gov.co/proteccionsocial/Paginas/R\%C3\%A9gimenContributivo.aspx, (3 de Junio de 2013).

Ministerio de Salud y Protección Social,Ministerio de Salud y Protección Social, Régimen Subsidiado. Obtenido de http://www.minsalud.gov.co/proteccionsocial/Paginas/R\%C3\%A9gimenSubsidiado.aspx, . (2 de Junio de 2013).

Montgomery, D.G.; Diseño y Análisis de Experimentos. Editorial Limusa S.A. De C.V. (2004).

Promodel; MEDmodel. Obtenido de http://www.promodel.com.mx/medmodel.php, (5 de marzo de 2014).

Ramwadhdoebe, S., Buskens, E., Sakkers, R., Stahl, J. E.; A Tutorial on Discrete-Event Simulation for Health Policy Design and Decision Making: Optimizing Pediatric Ultrasound Screening for Hipdysplasia as an Illustration, Health Policy, 143-150 (2009).

Shannon, E. R.; Simulación de Sistemas., 1를. Ed. Editorial Trillas,México, (1988).

Superintendencia Nacional de Salud. Costos de Servicio en Salud. Bogotá. (2010).

Thorwarth, M., y Arisha, A.; A Simulation-based Decision Support System to Model Complex Demand Driven Healthcare Facilities. Winter Simulation Conference. Berlin, (2012).

Unisalud; Informe de Gestion 2012. Bogotá, . (2012).

Valderrama, J. O.; Principales Aspectos sobre la Preparación de un artículo para ser Publicado en una Revista Internacional de Corriente Principal. Inf. Tecnol., 3-14, (2005).

Virtue, A., Chaussale, T., y Kelly, J.; Using Simplified Discrete-Event Simulation Models for Health Care Applications. Winter Simulation Conference, 1154-1165. Phoenix. (2011).

Young, T., Eastock, J., Jahangirian, M., Naseer, A., y Lilford, R.; Three Critical Challenger for Modeling and Simulation in Healthcare. Winter Simulation Conference, 1823-1830. Austin, (2009). 
